\title{
Dexmedetomidine alleviates blunt chest trauma and hemorrhagic shock-resuscitation-induced acute lung injury through inhibiting the NLRP3 inflammasome
}

TINGQIAN MING* ${ }^{*}$ MIN YUAN*, QIAN KONG, QIN HUANG, ZHONGYUAN XIA and XIAOJING WU

Department of Anesthesiology, Renmin Hospital, Wuhan University, Wuhan, Hubei 430060, P.R. China

Received November 28, 2019; Accepted June 12, 2020

DOI: $10.3892 / \mathrm{mmr} .2020 .11335$

\begin{abstract}
Blunt chest trauma with hemorrhagic shock frequently induces pulmonary inflammation that leads to acute lung injury (ALI). The present study aimed to explore the protective effects of dexmedetomidine (Dex) in blunt chest trauma and hemorrhagic shock-resuscitation (THSR)-induced ALI by mediating nucleotide binding and oligomerization domain-like receptor family pyrin domain-containing protein 3 (NLRP3) inflammasome formation in rats. An ALI model in rats induced by THSR was constructed and Dex was administered intraperitoneally $(5 \mu \mathrm{g} / \mathrm{kg} / \mathrm{h})$ immediately after blunt chest trauma. Blood samples were collected for the determination of proinflammatory factor levels, and lung tissue specimens were harvested for wet/dry (W/D) weight ratio, hematoxylin and eosin staining, and transmission electron microscopy analyses. Additionally, malondialdehyde (MDA), superoxide dismutase (SOD), lactate dehydrogenase (LDH) and myeloperoxidase (MPO) activity were evaluated, and the expression of protein in lung tissues was examined via western blot analysis. Compared with the sham group,
\end{abstract}

Correspondence to: Professor Zhongyuan Xia or Dr Xiaojing Wu, Department of Anesthesiology, Renmin Hospital, Wuhan University, 238 Liberation Road, Wuhan, Hubei 430060, P.R. China

E-mail: xiazhongyuan2005@aliyun.com

E-mail:wxj164@163.com

${ }^{*}$ Contributed equally

Abbreviations: ALI, acute lung injury; Dex, dexmedetomidine; THSR, blunt chest trauma and hemorrhagic shock-resuscitation; NLRP3, nucleotide binding and oligomerization domain-like receptor family pyrin domain-containing protein 3; ASC, apoptosisassociated speck-like protein containing a caspase recruitment domain; W/D, wet/dry; MDA, malondialdehyde; SOD, superoxide dismutase; LDH, lactate dehydrogenase; MPO, myeloperoxidase; ARDS, acute respiratory distress syndrome; IL, interleukin; TNF, tumor necrosis factor; HS, hemorrhagic shock; MAP, mean arterial pressure; VILI, ventilator-induced lung injury

Key words: NLRP3, inflammasome, dexmedetomidine, acute lung injury, trauma, inflammatory response pathological alterations in the ALI group and the W/D ratios were significantly increased. MDA, LDH and MPO activity, and the levels of interleukin (IL)-1 $\beta$, IL-18, IL-6 and tumor necrosis factor- $\alpha$ were significantly elevated. NLRP3, apoptosis-associated speck-like protein containing a caspase recruitment domain and caspase-1 expression was significantly increased. Conversely, Dex treatment significantly reversed these changes. The present study demonstrated that by reducing inflammatory responses, Dex exerted protective effects against THSR-ALI in rats, potentially via the inhibition of NLRP3 signaling pathways.

\section{Introduction}

Blunt chest trauma is commonly associated with a wide range of injuries, a number of which are life-threatening with high mortality and require immediate medical attention (1). Hemorrhagic shock (HS), which endangers patients' lives and causes a high trauma-induced mortality rate of 30-40\%, is a common complication in patients who suffer from blunt chest trauma (2,3). Furthermore, the ensuing hypoxemia and ischemia during HS, and the resulting ischemia-reperfusion injury following HS commonly aggravate tissue damage $(4,5)$. The lungs are frequently the first organ to become injured; acute lung injury (ALI) is the most common lung injury (6). ALI is secondary to HS in thoracic trauma and may lead to acute respiratory distress syndrome (ARDS) (6). An epidemiological study estimated that the annual prevalence of ARDS in the USA is 5-35 cases for every 100,000 individuals, depending on the definitions utilized and the design of study methodology (7). Therefore, the pathogenesis and pathophysiology of ALI/ARDS require elucidation to identify and develop novel targeted therapies for this disorder. ALI/ARDS is closely associated with oxidative stress and inflammatory responses; however, no widely available, effective and specific interventions have been established for ALI/ARDS treatment (7).

As an important mediator in HS and various types of ALI/ARDS $(8,9)$, the nucleotide binding and oligomerization domain-like receptor family pyrin domain-containing protein 3 (NLRP3) inflammasome is an oligomeric molecular complex composed of the NLRP3 scaffold, adapter protein apoptosis-associated speck-like protein (ASC) and caspase-1 (10). The NLRP3 inflammasome plays an important 
role in immune responses and disease processes such as type II diabetes (11), atherosclerosis (12) and inflammatory bowel disease (13). MCC950, which can block both canonical and non-canonical NLRP3 inflammasome activation, was reported to lower pulmonary inflammation in mice (14). Furthermore, activation of the NLRP3 inflammasome was demonstrated to be involved in excessive inflammatory responses, and to be closely associated with ALI/ARDS pathogenesis (15). Therefore, as an important component of innate immunity, the NLRP3 inflammasome may provide novel targets for the treatment of various inflammatory diseases.

Dexmedetomidine (Dex) is a short-acting, highly selective $\alpha-2$ adrenoreceptor agonist that is extensively applied in clinical anesthesia and intensive care $(16,17)$. A number of basic research experiments and clinical trials have reported that Dex can protect various organs, including the lungs, by inhibiting oxidative stress, inflammatory responses and apoptosis in a range of diseases (18-20). The protective effects of Dex were recently demonstrated in a two-hit rat model (21). A recent study has shown that Dex is relevant to the inhibition of NLRP3 inflammasome activity (19). Two recent studies reported that Dex can inhibit the NF- $\kappa$ B pathway and NLRP3 inflammasome to suppress the inflammatory response $(22,23)$. However, the question of whether Dex exerts protective effects on trauma-induced ALI has not been investigated, and the underlying molecular mechanisms remain unclear. Therefore, the present study focused on the NLRP3 inflammasome in order to elucidate the possible cellular and molecular events involving NLRP3 inflammasome in Dex-mediated alleviation of THSR-induced ALI in a rat model.

The present study aimed to test the hypothesis that Dex exerts protective effects on THSR-induced ALI in rats by alleviating the inflammatory response and inhibiting NLRP3 signaling pathways.

\section{Materials and methods}

Ethics statement. All laboratory and animal experiments were approved by the Medical Ethics Committee of Renmin Hospital of Wuhan University in accordance with the Guide for the Care and Use of Laboratory Animals of the National Research Council (US) Committee (24).

Animals. Healthy adult male Sprague-Dawley rats (age, 8 weeks; weight, 200-220 g) were obtained from Vital River Laboratory Animal Technology Co., Ltd. (certificate no. SYXK 2014-0080) and maintained under specific pathogen-free conditions $\left(12: 12\right.$-h light/dark cycle, $20-24^{\circ} \mathrm{C}$ and 40-60\% humidity) with food and water ad libitum.

Experimental protocols. A total of 40 rats were randomly divided into four equal groups: Sham, Dex, ALI and Dex + ALI groups. Rats in the ALI and Dex + ALI groups underwent THSR surgery, whereas those in the sham and Dex groups were subjected to sham surgery, including femoral arterial and venous cannulation, although neither blunt chest trauma nor HS-resuscitation were induced. In addition, rats in the Dex and Dex + ALI groups were administered with $5 \mu \mathrm{g} / \mathrm{kg} / \mathrm{h}$ Dex for $30 \mathrm{~min}$ via intraperitoneal injection (cat. no. 14030332; Jiangsu Hengrui Medicine Co., Ltd.) immediately after the sham or blunt chest traumatic surgery. At $6 \mathrm{~h}$ after the surgery, all animals were sacrificed under anesthesia via intraperitoneal injection of $50 \mathrm{mg} / \mathrm{kg}$ pentobarbital sodium and exsanguination from the right carotid artery. At the same time, arterial blood, bronchoalveolar lavage fluid and lung tissue samples were collected. Lung tissues were snap-frozen in liquid nitrogen and stored at $-80^{\circ} \mathrm{C}$ for subsequent analysis. All animal experiments were performed from 08:00 to 12:00.

Animal model of THSR-induced ALI. The rat model of THSR-induced ALI adopted in the present study was described in mice by Seitz et al (25). This model was successfully established in rats in our previous studies $(26,27)$.

In brief, the rats were provided free access to water and fasted for $12 \mathrm{~h}$ prior to surgery. After induction of anesthesia via intraperitoneal injection of pentobarbital sodium $(2 \%$; $50 \mathrm{mg} / \mathrm{kg}$ ), sham or THSR surgery was performed according to the groupings. A polyethylene tube filled with heparinized saline was used for cannulation of the femoral artery and vein to monitor continuous invasive pressure [in the form of mean arterial pressure (MAP)] and heart rate using a monitor (IntelliVue MP20; Philips Healthcare), as well as to establish venous access. As previously described, blunt chest trauma was induced with a fixed 2.45-J chest impact by dropping a hollow cylindrical encased in a vertical stainless steel tube which was positioned onto a lexon platform from a definite height (26). A precordial shield directed the impact force to the lungs bilaterally; thus, cardiac trauma was prevented. After $5 \mathrm{~min}$, blood was withdrawn into a heparinized syringe until the MAP reached 35-45 $\mathrm{mmHg}$ in $15 \mathrm{~min}$ to induce HS, and the MAP was maintained for $1 \mathrm{~h}$ by drawing blood via transfusion. Then, it was determined that the MAP met the criteria for HS as previously described (26). Furthermore, the rats were transfused with a 1:1 mix of all the withdrawn blood and Ringer's lactate solution (Baxter Healthcare Co., Ltd.) to induce resuscitation during the next $1 \mathrm{~h}$.

Blood gas analysis. At $6 \mathrm{~h}$ following surgery, blood samples were collected from animals under anesthesia from the right femoral artery $(0.5 \mathrm{ml} / \mathrm{animal})$ and then immediately assayed with an i-STAT portable clinical analyzer (Abbott Point Of Care, Inc.; Abbott Pharmaceutical Co., Ltd.). Arterial partial pressure of oxygen $\left(\mathrm{PaO}_{2}\right)$ was measured, and the oxygenation index $\left[\mathrm{PaO}_{2} /\right.$ fraction of inspired oxygen $\left.\left(\mathrm{FiO}_{2}\right)\right]$ was calculated.

Measurement of lung wet/dry (W/D) weight ratio. The W/D ratio is used as an index of the severity of pulmonary edema. The W/D ratio was determined by measuring the water content in the lungs $6 \mathrm{~h}$ after THSR challenge. The right middle lobe of the lungs was dissected from non-pulmonary tissues and then accurately weighed to determine wet weight using an electronic balance after the surface blood and water were wiped off. Afterward, the lungs were incubated for $72 \mathrm{~h}$ in an oven at $60^{\circ} \mathrm{C}$ and then reweighed to determine the dry weight. Finally, the W/D ratio was calculated.

Hematoxylin and eosin (H\&E) staining and lung injury score. Lung tissue samples were collected and fixed in $4 \%$ paraformaldehyde at $4^{\circ} \mathrm{C}$ for $48 \mathrm{~h}$. Subsequently, the samples were dehydrated, embedded in paraffin and sectioned routinely 
(5 $\mu \mathrm{m}$ thickness). Finally, H\&E staining was performed with the following steps at room temperature using H\&E solution (Sigma-Aldrich; Merck KGaA): Firstly, paraffin sections were incubated at $60^{\circ} \mathrm{C}$ for $30 \mathrm{~min}$, twice immersed in xylene for $15 \mathrm{~min}$ at room temperature and then treated with a descending ethanol series for $5 \mathrm{~min}$ each at room temperature. The sections were then treated with $0.5 \%$ hematoxylin for $1-5 \mathrm{~min}$ at room temperature and then rinsed in tap water for $1 \mathrm{~min}$. Sections were incubated with PBS for 8 sec until a blue color was observed, and then the sections were washed using tap water for $1 \mathrm{~min}$ and then distilled water for $8 \mathrm{sec}$. Sections were then stained with $1 \%$ eosin for $3 \mathrm{~min}$ at room temperature and then washed with tap water. Then, sections were treated with an ascending ethanol series for $1 \mathrm{~min}$ each at room temperature.

Pathological alterations of the lung tissues were observed under a light microscope (magnification, x200; BX51; Olympus Corporation). Simultaneously, lung injury scores were calculated by applying the histological scoring system designed for mice by Belperio et al (28). Histological scoring of 10 fields of each $5-\mu$ m paraffin-embedded tissue section was performed blindly for the following parameters: Congestion and hemorrhage of the alveoli; airway epithelial cellular damage; neutrophil infiltration or aggregation in airspace or vessel wall; and thickness of the alveolar wall or formation of hyaline membrane. These parameters were scored from grade 0 to 4 using specific criteria: Normal or little damage, 0 ; mild damage $(<25 \%),+1$; moderate damage $(25-50 \%),+2$; severe damage $(50-75 \%),+3$; and maximal damage $(>75 \%),+4$. The pathological score of each field was measured as the total score of all four items. The lung injury score of each section was expressed as the average value of the 10 fields.

Transmission electron microscopy. Fragments of the right middle lung tissues were cut into $1-\mathrm{mm}$ slices and then fixed in $2.5 \%$ glutaraldehyde solution for $2 \mathrm{~h}$ at $0-4^{\circ} \mathrm{C}$. Afterward, the specimens were rinsed with PBS three times, fixed with $1 \%$ osmic acid at room temperature for $1 \mathrm{~h}$, rinsed with PBS three times and stained with $2 \%$ uranium acetate solution at room temperature for $30 \mathrm{~min}$, and then dehydrated with dimethyl ketone. The specimens were embedded in Epon- 812 at $60^{\circ} \mathrm{C}$ for 2 days, cut into ultrathin sections $(60 \mathrm{~nm})$, and stained at room temperature with $1 \%$ uranyl acetate for $30 \mathrm{~min}$ and lead citrate for $15 \mathrm{~min}$. The sections were observed under a transmission electron microscope (magnification, x10,000; Hitachi H-600; Hitachi, Ltd.).

Measurement of malondialdehyde (MDA), superoxide dismutase (SOD), lactate dehydrogenase (LDH) and myeloperoxidase (MPO) activities in lung tissues. MDA reflects the degree of lipid peroxidation, whereas SOD protects cells from superoxide damage (29). LDH and MPO activities are reported as indices of intracellular injury and neutrophil accumulation, respectively (29). LDH, MPO, SOD and MDA activity levels were determined via colorimetry. In brief, $100 \mathrm{mg}$ frozen $\left(4^{\circ} \mathrm{C}\right)$ lung tissue was cut, weighed, homogenized and centrifuged at $1,500 \mathrm{x}$ g for $10 \mathrm{~min}$ at $4^{\circ} \mathrm{C}$. The supernatants were collected, and MDA, SOD, LDH and MPO activities were determined using MDA, SOD, LDH and MPO assay kits (MDA, cat. no. A003-1-2; SOD, cat. no. A001-3-2;
LDH, cat. no. A020-2-2; MPO, cat. no. A044-1-1; Nanjing Jiancheng Bioengineering Institute Co., Ltd.) according to their respective manufacturer's protocols.

ELISA determination of serum interleukin (IL)-1 $\beta, I L-18$, IL-6, and tumor necrosis factor (TNF)- $\alpha$ expression levels. The blood samples were immediately centrifuged at $1,500 \mathrm{xg}$ for $10 \mathrm{~min}$ at $4^{\circ} \mathrm{C}$ after collection to obtain the serum. The supernatant fluid was harvested and assayed using ELISA kits (IL-1 $\beta$, cat. no. RLB00; IL-18, cat. no. DY521-05; IL-6, cat. no. R6000B; TNF- $\alpha$, cat. no. RTA00; all R\&D Systems, Inc.) to measure the levels of proinflammatory cytokines in serum according to the manufacturer's protocols. The absorbance was measured at $450 \mathrm{~nm}$ using an ELISA reader (BioTek Instruments, Inc.).

Western blot analysis of NLRP3, ASC and caspase-1. The lung tissue samples were homogenized with RIPA lysis buffer $(25 \mathrm{mM}$ Tris-HCl,pH 7.6, $1 \%$ NP-40,0.5\% sodium deoxycholate and $0.1 \%$ SDS) supplemented with $1 \%$ PMSF. Lysates were sonicated and centrifuged at $3,000 \times \mathrm{g}$ at $4^{\circ} \mathrm{C}$ for $10 \mathrm{~min}$, and then the homogenate supernatant was centrifuged again at $10,000 \mathrm{x}$ g at $4^{\circ} \mathrm{C}$ for 10 min to obtain the final lung homogenate supernatant, which was used to detect proteins. The concentrations of the proteins were measured using a bicinchoninic acid protein assay kit. On the basis of the protein concentrations, an equal quantity of protein $(30 \mu \mathrm{g})$ was loaded into each well and then separated via $10 \%$ SDS-PAGE. The proteins were subsequently electrotransferred to PVDF membranes. The membranes were blocked with $5 \%$ fat-free milk at room temperature for $1 \mathrm{~h}$ and then incubated with different primary polyclonal antibodies (rabbit anti-rat) at $4^{\circ} \mathrm{C}$ overnight. The primary polyclonal antibodies used in the present study were NLRP3 (1:1,000; cat. no. ab214185; Abcam), ASC (1:1,000; cat. no. D2W8U; Cell Signaling Technology, Inc.), caspase-1 (1:300; cat. no. ab1872; Abcam) and GAPDH (1:1,000; cat. no. sc-137179; Santa Cruz Biotechnology, Inc.). Afterward, the membranes were incubated with secondary antibody (horseradish peroxidase-conjugated goat anti-rabbit IgG; 1:2,000; cat. no. 7074; Cell Signaling Technology, Inc.) for $1 \mathrm{~h}$ at room temperature. The immunoreactive protein bands were visualized by enhanced chemiluminescence (cat. no. 32132; Thermo Fisher Scientific, Inc.) with an Odyssey color infrared laser scan-imaging instrument (LI-COR Biosciences). The quantities of the target proteins were analyzed using Image Lab software (version 5.2.1; National Institutes of Health) and reported as the densitometric ratios between the target protein and GAPDH, which was used as a loading control.

Statistical analysis. All quantitative data are presented as the mean \pm SD ( $=10 /$ group). Multiple comparisons were performed for statistical analysis via one-way ANOVA by using GraphPad Prism Software 7.0 (GraphPad Software, Inc.). Bonferroni post hoc test was used to test differences between individual means when the F-statistic was significant. $\mathrm{P}<0.05$ was considered to indicate a statistically significant difference.

\section{Results}

Effects of Dex on $\mathrm{PaO}_{2}$ and $\mathrm{PaO}_{2} / \mathrm{FiO}_{2}$ in THSR-induced ALI rats. The results of arterial blood gas analysis revealed that 

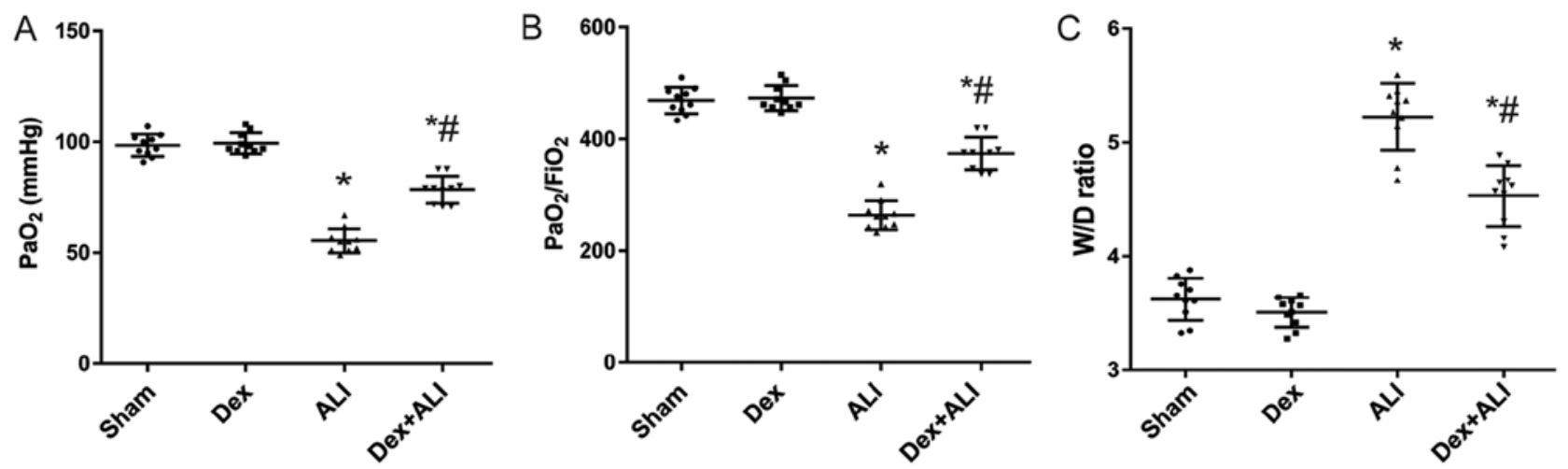

Figure 1. Dex attenuates the effects of THSR-induced ALI on arterial blood gas. Dex increases the levels of (A) $\mathrm{PaO}_{2}$ and (B) $\mathrm{PaO}_{2} / \mathrm{FiO}_{2}$, and decreased (C) lung W/D ratios in a rat model of THSR-induced ALI. Data are presented as the mean $\pm \mathrm{SD}(\mathrm{n}=10)$. " $\mathrm{P}<0.05$ vs. sham; " $\mathrm{P}<0.05$ vs. ALI. ALI, acute lung injury; Dex, dexmedetomidine; $\mathrm{PaO}_{2}$, partial pressure of oxygen; $\mathrm{FiO}_{2}$, fraction of inspired oxygen; W/D, wet/dry; THSR, blunt chest trauma and hemorrhagic shock-resuscitation.

the levels of $\mathrm{PaO}_{2}$ significantly decreased in the ALI group $6 \mathrm{~h}$ after THSR procedure (Fig. 1A). Furthermore, the $\mathrm{PaO}_{2} / \mathrm{FiO}_{2}$ ratios in the ALI group significantly decreased significantly (Fig. 1B). Dex treatment significantly attenuated the decreases induced by THSR challenge (Fig. 1A and B).

Dex decreases the lung W/D ratio in THSR-induced ALI. The lung W/D ratios in the ALI group were significantly increased compared with those in the sham group. Dex treatment significantly reversed the increase induced by THSR (Fig. 1C).

Dex attenuates lung pathological alterations in THSR-induced $A L I$. H\&E staining of the lung sections in the sham group showed parenchymal microscopic findings of normal lungs: The alveolar structure of the sham group was intact, the alveolar wall was smooth and the pulmonary interstitial showed no evident exudation (Fig. 2A). No notable changes were observed in the Dex group. However, the ALI group showed a notable disruption to normal alveolar structure, severe alveolar congestion, hemorrhage, rupture, and infiltration with exuded and accumulated inflammatory cells; the intervals of lung tissues were markedly widened and thickened, and pulmonary interstitial edema and alveolar cavity fusion were also shown. By contrast, the Dex + ALI group exhibited relatively mild intra-alveolar hemorrhage, infiltration of inflammatory cells, pulmonary interstitial edema, and narrower alveolar intervals (Fig. 2A).

Lung injury scores were calculated to indicate the lung pathological alterations based on the observations of lung damage parameters under a light microscope. The ALI group reported significantly higher lung injury scores than the sham group. Conversely, the Dex + ALI group exhibited significantly reduced lung injury scores compared with the ALI group (Fig. 2B).

Transmission electron microscopy was used to examine the ultrastructural changes in lung tissues. Electron microscopy of the lung tissues in the sham or Dex group demonstrated clear edges of cells, nuclear membranes and certain osmiophilic lamellar bodies, whereas the ALI group showed significant pathological alterations and decreased number of lamellar bodies. Moreover, the Dex + ALI group exhibited a substantial attenuation of THSR-induced pulmonary injury compared with the ALI group (Fig. 3).
Dex decreases $L D H$ levels, $M D A$ and MPO activity, and increases SOD activity to inhibit THSR-induced ALI. Oxidative stress indices (MPO, SOD, LDH and MDA) were selected to determine ALI severity and explore the mechanisms of THSR-induced ALI. As presented in Fig. 4, MDA, LDH and MPO levels in the ALI group were significantly increased compared with those in the sham group. Dex treatment resulted in significant decreases compared with the ALI group (Fig. 4A, C and D). By contrast, SOD activity was significantly increased in the ALI group compared with the sham group, which was attenuated by Dex (Fig. 4B).

Dex inhibits inflammatory responses by decreasing serum $I L-1 \beta, I L-18, I L-6$ and TNF- $\alpha$ levels in THSR-induced ALI. The serum expression levels of the proinflammatory cytokines IL-1 $\beta$, IL-18, IL-6 and TNF- $\alpha$ in the ALI group were significantly increased compared with those in the sham group. Conversely, Dex treatment resulted in a significant decrease in their expression levels compared with the ALI group (Fig. 5A-D).

Dex attenuates THSR-induced ALI by upregulating NLRP3, $A S C$, and caspase-1 protein expression in lung tissues. Western blot analysis was performed to determine NLRP3, ASC and caspase-1 protein expression levels (Fig. 6A). Following THSR, NLRP3, ASC and caspase-1 levels were significantly increased compared with the sham group. Dex treatment significantly decreased NLRP3, ASC and caspase-1 protein expression levels in ALI model rats (Fig. 6B).

\section{Discussion}

In a previous study, a rat model of ALI was successfully established using THSR (26). We did not induce HS by purely referring to the relative blood volume loss per kilogram because blunt chest trauma itself could exert a considerable effect on blood pressure, which also contributes to the induction of HS. This hypothesis was confirmed in a model of blunt chest trauma in rats (30). The results of the present study were consistent with those of previous studies $(25-27,30)$. The findings indicated that the rat ALI model was successfully established based on the arterial blood gases, which were improved by Dex in the 

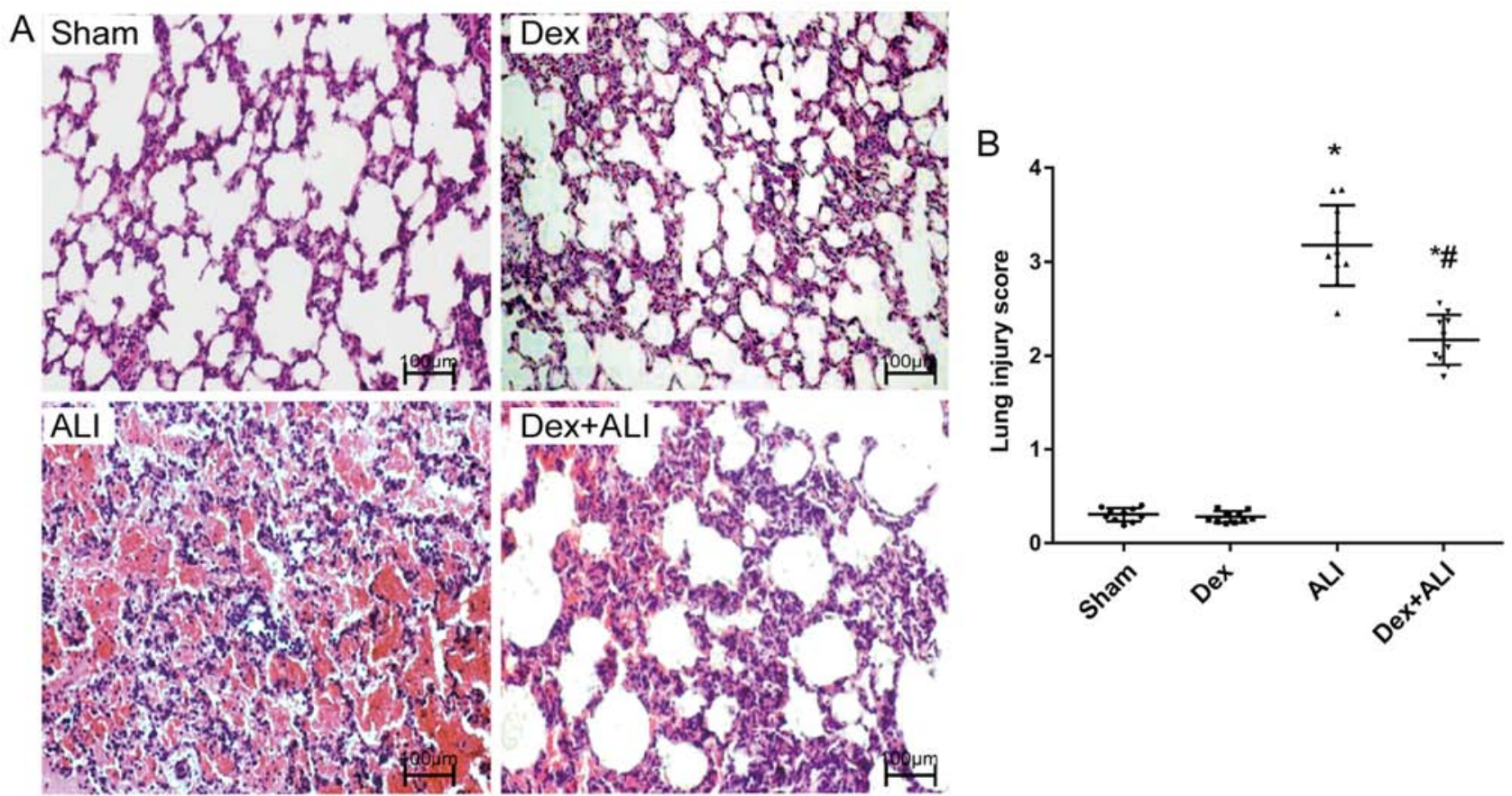

Figure 2. Dex attenuates lung pathological alterations in blunt chest trauma and hemorrhagic shock-resuscitation-induced ALI. (A) Microscopic presentations of lung tissues by H\&E staining (magnification, $x 400$ ). (B) Detection of lung injury measured by the scoring system of lung injury. Data are presented as the mean $\pm \mathrm{SD}(\mathrm{n}=10) .{ }^{*} \mathrm{P}<0.05$ vs. sham; ${ }^{*} \mathrm{P}<0.05$ vs. ALI. ALI, acute lung injury; Dex, dexmedetomidine.
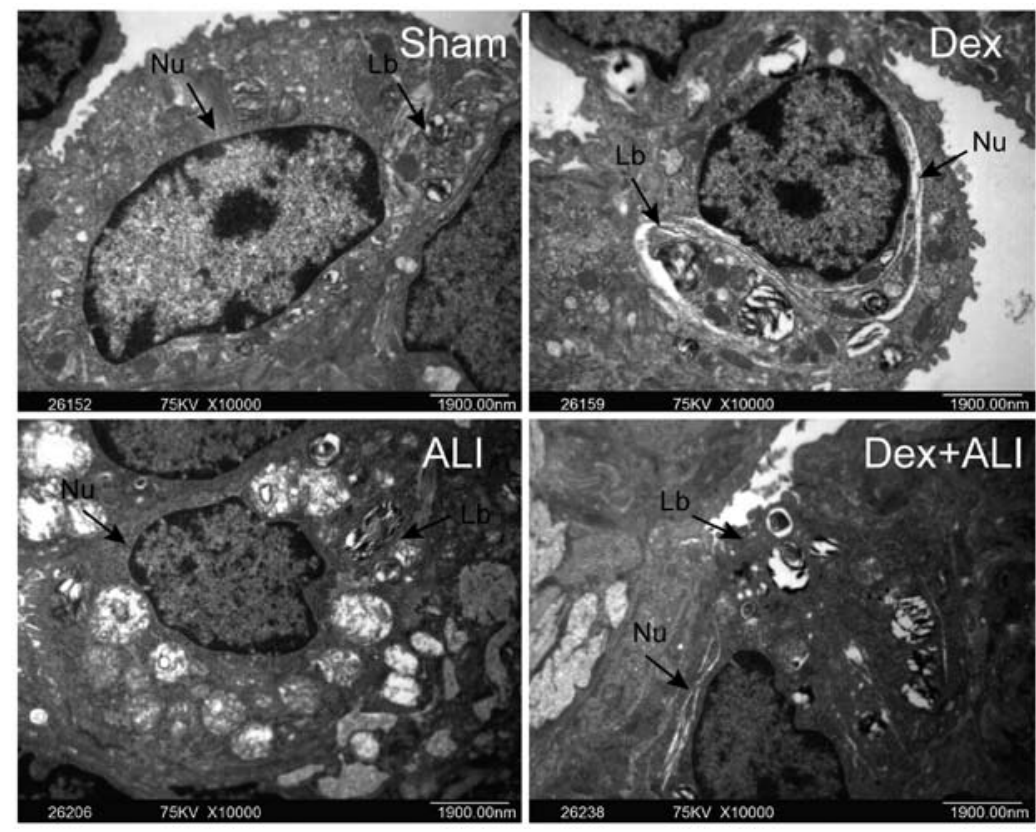

Figure 3. Dex attenuates pathological alterations to the lungs in THSR-induced ALI. Lung pathology was evaluated in a rat model of THSR-induced ALI via transmission electron microscopy (magnification, x10,000). ALI, acute lung injury; Dex, dexmedetomidine; THSR, blunt chest trauma and hemorrhagic shock-resuscitation; Lb, lamellar body; Nu, nucleus.

rat model of THSR-induced ALI. Additionally, the lung W/D ratios showed the severity of pulmonary edema in different groups; the results showed that the condition of pulmonary edema was worse in the THSR-induced ALI group, and that treatment of Dex alleviated the severity of pulmonary edema in ALI rats. As for the condition of the lungs, the ALI group showed extensive pulmonary injury, whereas Dex ameliorated pathological alterations to the lung in the model.
Severe THSR damage often triggers systemic inflammatory response syndrome and multiple organ dysfunction syndrome (1). ALI or ARDS are common pathophysiological processes due to 'waterfall' inflammatory reactions resulting from traumatic pulmonary contusion $(1,31)$. Lung tissue damage is associated with inflammatory reactions, particularly when infection secondary to trauma plays an important role in the process of ALI; the production of oxygen radicals 

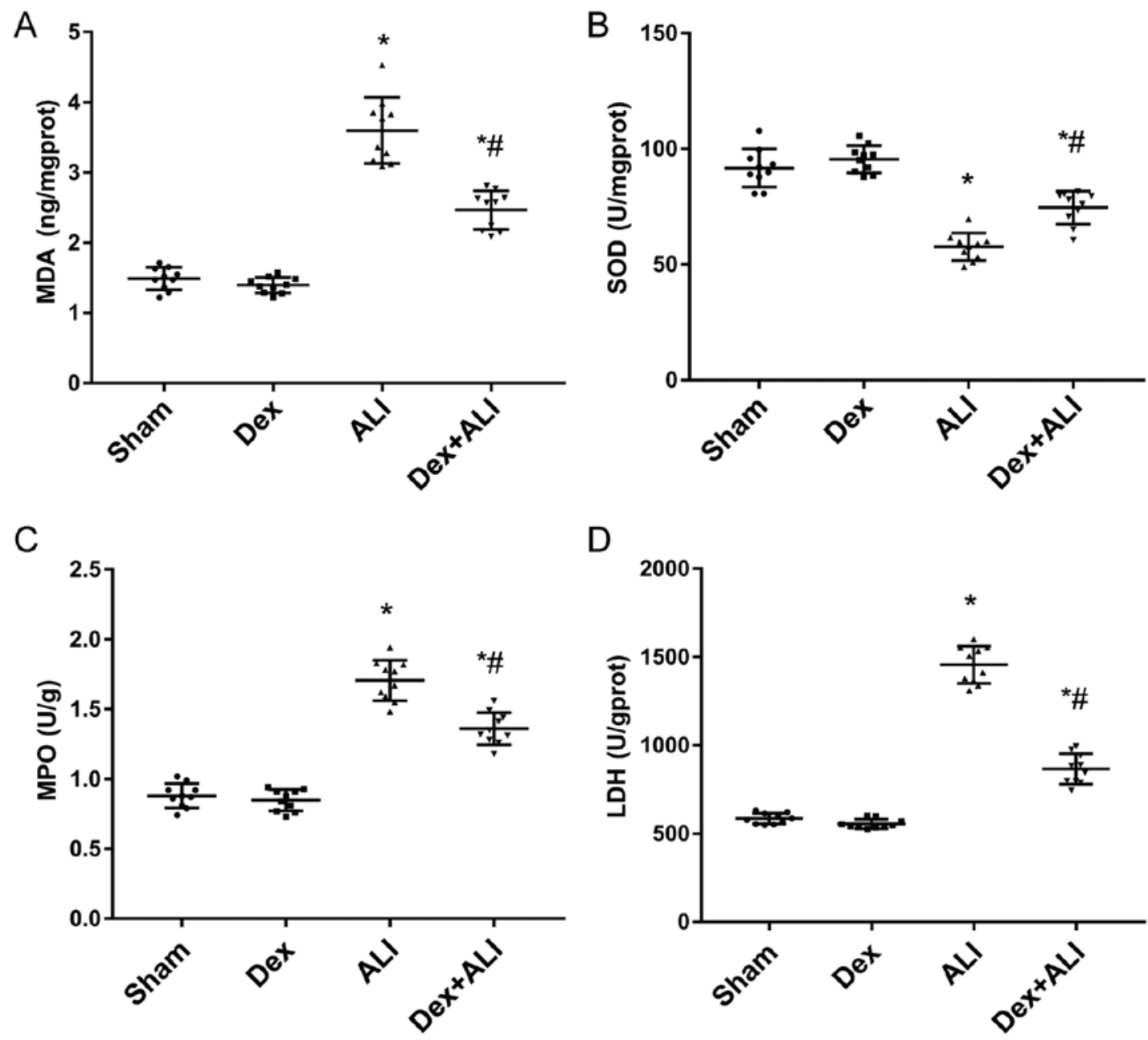

Figure 4. Effects of Dex treatment on oxidative stress markers in a rat model of THSR-induced ALI. (A) Level of MDA and activity of (B) SOD, (C) MPO and (D) LDH in THSR-induced ALI. Data are presented as the mean $\pm \mathrm{SD}(\mathrm{n}=10)$. ${ }^{*} \mathrm{P}<0.05$ vs. sham; ${ }^{\text {P }}<0.05$ vs. ALI. ALI, acute lung injury; Dex, dexmedetomidine; THSR, blunt chest trauma and hemorrhagic shock-resuscitation; MDA, malondialdehyde; SOD, superoxide dismutase; LDH, lactate dehydrogenase; MPO, myeloperoxidase.

by the respiratory burst of granulocytes or monocytes is also an important part of defense processes (32). MDA, LDH and MPO activity levels reflect the degree of cell injury and inflammatory responses, whereas SOD is a pivotal antioxidant enzyme reflecting the ability to scavenge the oxygen-free radicals and protects cells from superoxide damage (29). The measurement of SOD activity and MDA levels in lung tissue revealed the balance between antioxidant action and oxidation. As observed in previous studies $(32,33)$, oxidative system activation and excessive free radical production were observed, but the antioxidant system was partially destroyed and deactivated in response to trauma, which were demonstrated by detection of MDA and SOD levels. In the present study, THSR-induced ALI increased MDA levels and decreased SOD activity in lung tissue; at the same time, the expression levels of the proinflammatory cytokines IL-1 $\beta$, IL-18, IL-6 and TNF- $\alpha$ were significantly increased in the ALI group. However, treatment with Dex alleviated the degree of oxidative stress and improved the antioxidant reaction to the overproduction of oxygen radicals in the experimental model.

As the core of innate immunity, NLRP3 forms an oligomeric molecular complex called an inflammasome, which responds to harmful stimuli, such as pathogen-associated molecular patterns and danger-associated molecular patterns (34). After NLRP3 activation, ASC is recruited, caspase-1 is activated, and pro-IL-1 $\beta$ and pro-IL-18 are processed into mature IL-1 $\beta$ and IL-18, respectively (34). These cytokines are released to induce selective recruitment of monocytes, neutrophils and lymphocytes, eventually causing pyroptosis, a novel form of programed cell death $(34,35)$, which leads to tissue damage as a consequence. ALI/ARDS pathogenesis is closely associated with the NLRP3 inflammasome in lipopolysaccharide (LPS)-induced mouse and macrophage models (36), in LPS-induced animal models of ALI $(36,37)$, and in cecal ligation perforation (38). The present study demonstrated that NLRP3, ASC and caspase-1 expression levels were elevated in the ALI group, which was consistent with the aforementioned studies. These results suggested that the NLRP3 pathway was activated in THSR-induced ALI, and that Dex inhibited the NLRP3 inflammasome in this model, indicating that NLRP3 inflammasome signaling activation may serve an important role in ALI.

An increasing number of studies have reported that Dex exerts protective effects on the lungs $(18,39-41)$. A recent study discovered that Dex alleviates multiple organ damage 

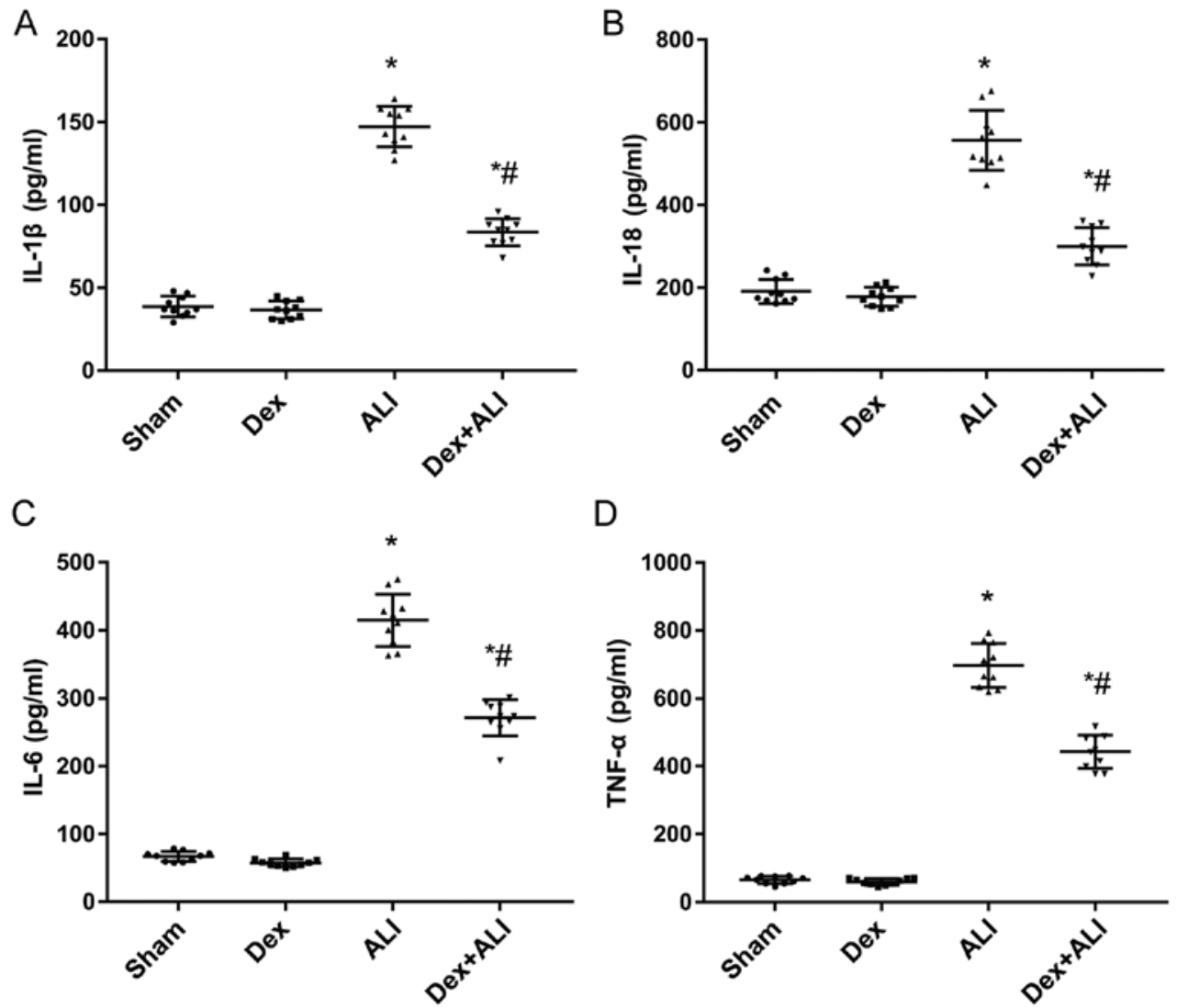

Figure 5. Effects of Dex treatment on cytokine levels in a rat model of THSR-induced ALI. (A) IL-1 $\beta$, (B) IL-18, (C) IL-6 and (D) TNF- $\alpha$ levels in the serum of THSR-induced ALI rats. Data are presented as the mean $\pm \mathrm{SD}(\mathrm{n}=10)$. "P $<0.05$ vs. sham; ${ }^{*} \mathrm{P}<0.05$ vs. ALI. ALI, acute lung injury; Dex, dexmedetomidine; THSR, blunt chest trauma and hemorrhagic shock-resuscitation; IL, interleukin; TNF, tumor necrosis factor.

A

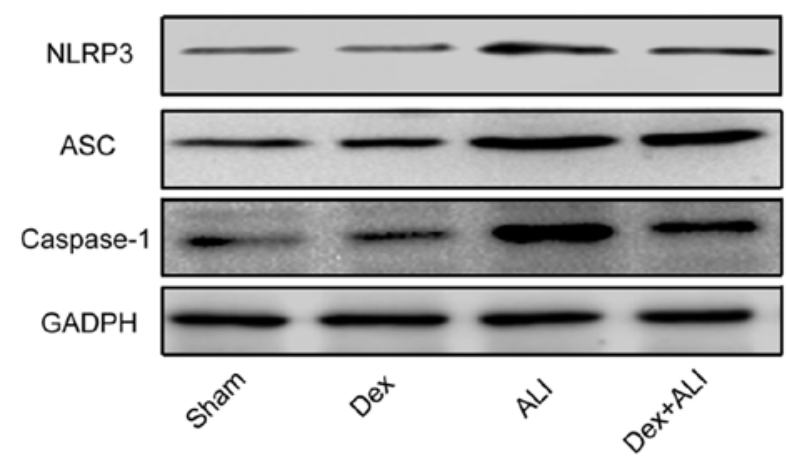

B

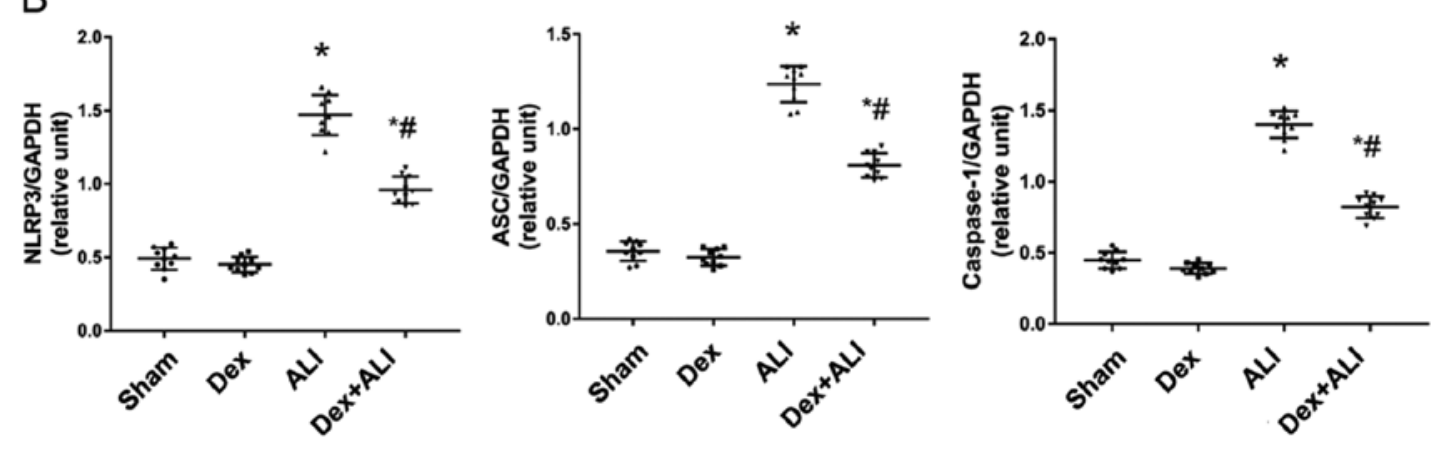

Figure 6. Dex treatment inhibits the activation of NLRP3 signaling pathways in a rat model of THSR-induced ALI. (A) Protein expression levels of NLRP3, ASC and caspase-1 in the lung tissue of THSR-induced ALI rats were determined via western blot analysis. GAPDH was used as a loading control. (B) Protein expression was quantified and expressed as densitometric ratios between the target protein and GAPDH. Data are presented as the mean \pm SD ( $n=10$ ). ${ }^{*} \mathrm{P}<0.05$ vs. sham; ${ }^{\mathrm{P}}<0.05$ vs. ALI. ALI, acute lung injury; Dex, dexmedetomidine; THSR, blunt chest trauma and hemorrhagic shock-resuscitation; NLRP3, nucleotide binding and oligomerization domain-like receptor family pyrin domain-containing protein 3; ASC, apoptosis-associated speck-like protein containing a caspase recruitment domain. 
by inhibiting the inflammatory response and oxidative stress in a two-hit hemorrhagic, resuscitation and subsequent endotoxemia model in rats; these effects were partially mediated by $\alpha 2$-adrenoceptors (20). Based on these findings, the effects of Dex on the lungs were investigated in a rat model of THSR-induced ALI. Another study found that Dex inhibits the NLRP3 inflammasome and sympathetic nerve activity in mice with acute pancreatitis (42). However, the underlying mechanisms remain unclear. A previous study reported that clinically relevant doses of Dex do not significantly affect the attenuation of ventilator-induced lung injury (VILI) (43). However, Dex at a dose that is $\sim 10$-fold higher than the recommended clinical dose had a significant effect on VILI attenuation $(43,44)$. On the basis of these findings, $5.0 \mu \mathrm{g} / \mathrm{kg} / \mathrm{h}$ was used as the dose of Dex in the present study to explore the underlying mechanisms. The findings of the present study indicated that Dex improved arterial blood gas levels, alleviated the severity of pulmonary edema, ameliorated pathological lung alterations, attenuated inflammatory responses, alleviated the degree of oxidative stress and inhibited the NLRP3 inflammasome signaling pathway at the same time. These results confirmed that Dex treatment may contribute to exert protective effects on THSR-induced ALI in rats.

However, the present study contains several limitations. The regulatory mechanism of NLRP3 inflammasome activation and the role of Dex in this mechanism remain unclear. Moreover, the question of whether Dex directly interacts with the NLRP3 inflammasome or via other indirect mechanisms that trigger signaling remains unanswered. Furthermore, there remain unresolved issues regarding whether the interaction was through the $\alpha 2$ adrenergic receptor pathway and whether other drugs aside from Dex could exert similar protective effects. If so, whether these effects were different, and whether the NLRP3 inflammasome plays the same role in ALI attenuation by different drugs, require further verification. Further studies are required to address these issues and questions.

The present study demonstrated that Dex inhibits NLRP3 inflammasome signaling pathways and alleviates THSR-induced ALI in rats. This pathomechanism needs to be further analyzed in mechanistic and dose-related studies.

\section{Acknowledgements}

Not applicable.

\section{Funding}

The present study was supported by grants from the National Natural Science Foundation of China (grant nos. 81901952 and 81970722).

\section{Availability of data and materials}

All data generated or analyzed during this study are included in this published article.

\section{Authors' contributions}

TQM and MY drafted the manuscript. TQM and QK analyzed and interpreted the data and all manuscript figures. MY and
$\mathrm{QH}$ performed the transmission electron microscopy and lung injury score analysis. ZYX and XJW conceived and designed the study, reviewed the data and revised the manuscript. All authors read and approved the final manuscript.

\section{Ethics approval and consent to participate}

Ethical approval was provided by the Medical Ethics Committee of Renmin Hospital of Wuhan University. All surgical procedures were performed in accordance with the Guide for the Care and Use of Laboratory Animals of the National Research Council (US) Committee.

\section{Patient consent for publication}

Not applicable.

\section{Competing interests}

The authors declare that they have no competing interests.

\section{References}

1. Ehrnthaller C, Flierl M, Perl M, Denk S, Unnewehr H, Ward PA, Radermacher P, Ignatius A, Gebhard F, Chinnaiyan A and Huber-Lang M: The molecular fingerprint of lung inflammation after blunt chest trauma. Eur J Med Res 20: 70, 2015.

2. Kauvar DS and Wade CE: The epidemiology and modern management of traumatic hemorrhage: US and international perspectives. Crit Care 9 Suppl 5 (Suppl 5): S1-S9, 2005.

3. Sauaia A, Moore EE, Johnson JL, Chin TL, Banerjee A, Sperry JL, Maier RV and Burlew CC: Temporal trends of postinjury multiple-organ failure: Still resource intensive, morbid, and lethal. J Trauma Acute Care Surg 76: 582-593, 2014.

4. Angele MK, Schneider CP and Chaudry IH: Bench-to-bedside review: Latest results in hemorrhagic shock. Crit Care 12: 218 , 2008.

5. Eltzschig HK and Eckle T: Ischemia and reperfusion-from mechanism to translation. Nat Med 17: 1391-1401, 2011.

6. Shah CV, Localio AR, Lanken PN, Kahn JM, Bellamy S, Gallop R, Finkel B, Gracias VH, Fuchs BD and Christie JD: The impact of development of acute lung injury on hospital mortality in critically ill trauma patients. Crit Care Med 36: 2309-2315, 2008.

7. Villar J, Blanco J and Kacmarek RM: Current incidence and outcome of the acute respiratory distress syndrome. Curr Opin Crit Care 22: 1-6, 2016.

8. Xu P, Wen Z, Shi X, Li Y, Fan L, Xiang M, Li A, Scott MJ, Xiao G, Li S, et al: Hemorrhagic shock augments Nlrp3 inflammasome activation in the lung through impaired pyrin induction. J Immunol 190: 5247-5255, 2013.

9. Grailer JJ, Canning BA, Kalbitz M, Haggadone MD, Dhond RM, Andjelkovic AV, Zetoune FS and Ward PA: Critical role for the NLRP3 inflammasome during acute lung injury. J Immunol 192: 5974-5983, 2014.

10. Mangan MSJ, Olhava EJ, Roush WR, Seidel HM, Glick GD and Latz E: Targeting the NLRP3 inflammasome in inflammatory diseases. Nat Rev Drug Discov 17: 588-606, 2018.

11. Lee HM, Kim JJ, Kim HJ, Shong M, Ku BJ and Jo EK: Upregulated NLRP3 inflammasome activation in patients with type 2 diabetes. Diabetes 62: 194-204, 2013.

12. Duewell P, Kono H, Rayner KJ, Sirois CM, Vladimer G, Bauernfeind FG, Abela GS, Franchi L, Nuñez G, Schnurr M, et al: NLRP3 inflammasomes are required for atherogenesis and activated by cholesterol crystals. Nature 464: 1357-1361, 2010.

13. Zhen Y and Zhang H: NLRP3 inflammasome and inflammatory bowel disease. Front Immunol 10: 276, 2019.

14. Primiano MJ, Lefker BA, Bowman MR, Bree AG, Hubeau C, Bonin PD, Mangan M, Dower K, Monks BG, Cushing L, et al: Efficacy and pharmacology of the NLRP3 inflammasome inhibitor CP-456,773 (CRID3) in murine models of dermal and pulmonary inflammation. J Immunol 197: 2421-2433, 2016. 
15. Mizushina Y, Karasawa T, Aizawa K, Kimura H, Watanabe S, Kamata R, Komada T, Mato N, Kasahara T, Koyama S, et al: Inflammasome-independent and atypical processing of IL-1 $\beta$ contributes to acid aspiration-induced acute lung injury. J Immunol 203: 236-246, 2019.

16. Afonso J and Reis F: Dexmedetomidine: Current role in anesthesia and intensive care. Rev Bras Anestesiol 62: 118-133, 2012

17. Carollo DS, Nossaman BD and Ramadhyani U: Dexmedetomidine: A review of clinical applications. Curr Opin Anaesthesiol 21: 457-461, 2008.

18. Xu Y,Zhang R, Li C, Yin X, Lv C, Wang Y, Zhao W and Zhang X: Dexmedetomidine attenuates acute lung injury induced by lipopolysaccharide in mouse through inhibition of MAPK pathway. Fundam Clin Pharmacol 29: 462-471, 2015.

19. Zhang Q, Wu D, Yang Y, Liu T and Liu H: Dexmedetomidine alleviates hyperoxia-induced acute lung injury via inhibiting NLRP3 inflammasome activation. Cell Physiol Biochem 42: 1907-1919, 2017.

20. Kang K, Gao Y, Wang SC, Liu HT, Kong WL, Zhang X, Huang R, Qi ZD, Zheng JB, Qu JD, et al: Dexmedetomidine protects against lipopolysaccharide-induced sepsis-associated acute kidney injury via an $\alpha 7 \mathrm{nAChR}$-dependent pathway. Biomed Pharmacother 106: 210-216, 2018.

21. Jiang Y, Xia M, Huang Q, Ding D, Li Y, Zhang Z and Zhang X Protective effect of dexmedetomidine against organ dysfunction in a two-hit model of hemorrhage/resuscitation and endotoxemia in rats. Braz J Med Biol Res 52: e7905, 2019.

22. Cheng F, Yan FF, Liu YP, Cong Y, Sun KF and He XM: Dexmedetomidine inhibits the NF- $\mathrm{B}$ pathway and NLRP3 inflammasome to attenuate papain-induced osteoarthritis in rats. Pharm Biol 57: 649-659, 2019.

23. Yin D, Zhou S, Xu X, Gao W, Li F, Ma Y, Sun D, Wu Y, Guo Q, Liu H, et al: Dexmedetomidine attenuated early brain injury in rats with subarachnoid haemorrhage by suppressing the inflammatory response: The TLR4/NF- $\mathrm{BB}$ pathway and the NLRP3 inflammasome may be involved in the mechanism. Brain Res 1698: 1-10, 2018.

24. National Research Council (US) Committee for the Update of the Guide for the Care and Use of Laboratory Animals. Guide for the Care and Use of Laboratory Animals, 8th edition. National Academies Press (US), Washington (DC), 2011.

25. Seitz DH, Perl M, Liener UC, Tauchmann B, Braumüller ST, Brückner UB, Gebhard F and Knöferl MW: Inflammatory alterations in a novel combination model of blunt chest trauma and hemorrhagic shock. J Trauma 70: 189-196, 2011.

26. Wu XJ, Liu HM, Song XM, Zhao B, Leng Y, Wang EY, Zhan LY, Meng QT and Xia ZY: Penehyclidine hydrochloride inhibits TLR4 signaling and inflammation, and attenuates blunt chest trauma and hemorrhagic shock-induced acute lung injury in rats Mol Med Rep 17: 6327-6336, 2018.

27. Wu X, Song X, Li N,Zhan L, Meng Q and Xia Z: Protective effects of dexmedetomidine on blunt chest trauma-induced pulmonary contusion in rats. J Trauma Acute Care Surg 74: 524-530, 2013.

28. Belperio JA, Keane MP, Burdick MD, Londhe V, Xue YY, Li K, Phillips RJ and Strieter RM: Critical role for CXCR2 and CXCR2 ligands during the pathogenesis of ventilator-induced lung injury. J Clin Invest 110: 1703-1716, 2002.

29. Sun K, Fan J and Han J: Ameliorating effects of traditional Chinese medicine preparation, Chinese materia medica and active compounds on ischemia/reperfusion-induced cerebral microcirculatory disturbances and neuron damage. Acta Pharm Sin B 5: 8-24, 2015.
30. Kao RL, Huang W, Martin CM and Rui T: The effect of aerosolized indomethacin on lung inflammation and injury in a rat model of blunt chest trauma. Can J Surg 61: S208-S218, 2018.

31. Rendeki S and Molnár TF: Pulmonary contusion. J Thorac Dis 11 (Suppl 2): S141-S151, 2019.

32. Liu X and Chen Z: The pathophysiological role of mitochondrial oxidative stress in lung diseases. J Transl Med 15: 207, 2017.

33. Torun AC, Tutuncu S, Ustun B and Akdemir HU: A study of the therapeutic effects of resveratrol on blunt chest trauma-induced acute lung injury in rats and the potential role of endocan as a biomarker of inflammation. Inflammation 40: 1803-1810, 2017.

34. Lamkanfi $M$ and Dixit VM: Mechanisms and functions of inflammasomes. Cell 157: 1013-1022, 2014.

35. Pedraza-Alva G, Pérez-Martínez L, Valdez-Hernández L, Meza-Sosa KF and Ando-Kuri M: Negative regulation of the inflammasome: Keeping inflammation under control. Immunol Rev 265: 231-257, 2015.

36. Zhang Y, Li X, Grailer JJ, Wang N, Wang M, Yao J, Zhong R, Gao GF, Ward PA, Tan DX and Li X: Melatonin alleviates acute lung injury through inhibiting the NLRP3 inflammasome. J Pineal Res 60: 405-414, 2016.

37. Zhang H, Chen S, Zeng M, Lin D, Wang Y, Wen X, Xu C, Yang L, Fan X, Gong Y, et al: Apelin-13 administration protects against LPS-induced acute lung injury by inhibiting NF- $\kappa \mathrm{B}$ pathway and NLRP3 inflammasome activation. Cell Physiol Biochem 49: 1918-1932, 2018.

38. Li Z, Jia Y, Feng Y, Cui R, Miao R, Zhang X, Qu K, Liu C and Zhang J: Methane alleviates sepsis-induced injury by inhibiting pyroptosis and apoptosis: In vivo and in vitro experiments. Aging (Albany NY) 11: 1226-1239, 2019.

39. Deletombe B, Trouve-Buisson T, Godon A, Falcon D, Giorgis-Allemand L, Bouzat P, Bosson JL and Payen JF: Dexmedetomidine to facilitate non-invasive ventilation after blunt chest trauma: A randomised, double-blind, crossover, placebo-controlled pilot study. Anaesth Crit Care Pain Med 38: 477-483, 2019.

40. Fu C, Dai X, Yang Y, Lin M, Cai Y and Cai S: Dexmedetomidine attenuates lipopolysaccharide-induced acute lung injury by inhibiting oxidative stress, mitochondrial dysfunction and apoptosis in rats. Mol Med Rep 15: 131-138, 2017.

41. Meng L, Li L, Lu S, Li K, Su Z, Wang Y, Fan X, Li X and Zhao G: The protective effect of dexmedetomidine on LPS-induced acute lung injury through the HMGB1-mediated TLR4/NF- $\kappa \mathrm{B}$ and PI3K/Akt/mTOR pathways. Mol Immunol 94: 7-17, 2018.

42. Li Y, Pan Y, Gao L, Lu G, Zhang J, Xie X, Tong Z, Li B, Li G and Li W: Dexmedetomidine attenuates pancreatic injury and inflammatory response in mice with pancreatitis by possible reduction of NLRP3 activation and up-regulation of NET expression. Biochem Biophys Res Commun 495: 2439-2447, 2018.

43. Yang CL, Tsai PS and Huang CJ: Effects of dexmedetomidine on regulating pulmonary inflammation in a rat model of ventilatorinduced lung injury. Acta Anaesthesiol Taiwan 46: 151-159, 2008.

44. Tasdogan M, Memis D, Sut N and Yuksel M: Results of a pilot study on the effects of propofol and dexmedetomidine on inflammatory responses and intraabdominal pressure in severe sepsis. J Clin Anesth 21: 394-400, 2009.

This work is licensed under a Creative Commons Attribution-NonCommercial-NoDerivatives 4.0 International (CC BY-NC-ND 4.0) License. 